the mean of a normal distribution lies $0.03 \sigma$ above the boundary separating the lower 48.99 per cent. from the rest and $I \cdot 22 \sigma$ below the boundary separating the upper I I.06 per cent. from the rest. The six-bristle class thus occupies a region of the distribution of bristle numbers which would be subtended by $1 \cdot 25^{\sigma}$ if the curve were a normal one.

My table $I$ is drawn up to show the distribution of females in each generation together with the width in $\sigma$ of each bristle class. In order to estimate the width of the eight-bristle class it is necessary to assume in each generation but the last that the next fly counted would have had nine bristles. The estimate is an estimate of the minimum size of the class in all but the last generation. It is true that in the seventh and eighth generation the width of the eight class increases to $I \cdot 30 \sigma$ and $I \cdot 53 \sigma$. This may be compared to the six-bristle class in generation three and four in which the width is $1.25^{\circ}$ and I $37 \sigma$. In generation nine the width of the eight class falls to $0.59 \sigma$ and there is every reason to believe that if sufficient flies could have been counted the width of the eight class would have been about $0.8 \sigma$ as it was in four different counts I made (Rendel, I963).

There is no evidence of any threshold or canalisation comparable to that at four, which class spans about 6.0\% (Sheldon, Rendel and Finlay, I 964). The reason why only one fly with nine bristles has appeared by generation nine whereas flies with eight appeared in generation two seems to be that selection has increased bristle number over the whole population by little more than $0.5 \sigma$ which is not more than half the width of a bristle class. There is no reason for assuming that the eight class is any more canalised than any other class.

\title{
REFERENCES
}

PARSHAD, R., AND NARDA, R. D. 1964. Canalisation and threshold effect of the extra scutellar phenotype in Drosophila melanogaster. Heredity, rg, 334-335.

RENDEL, J. M. 1963. Correlation between the number of scutellar and abdominal bristles in Drosophila melanogaster. Genetics, 48, 391 .

SHELDON, B. L., RENDEL, J. M., AND FINLAY, D. E. 1964. The effect of homozygosity on developmental stability. Genetics, 49, 471 .

\section{UNSTABLE MUTATION IN NEUROSPORA}

G. W. GRIGG

Division of Animol Genetics, C.S.I.R.O., P.O. Box 90, Epping, Sydney, N.S.W.

Received I.x.64

\section{INTRODUCTION}

In experiments involving ultraviolet irradiation of a histidine (his-) strain of Neurospora, many of the prototrophic colonies which arose were no longer his ${ }^{+}$when later tested. This note describes their properties.

\section{PROCEDURE}

Uninucleate microconidia (and in one experiment macroconidia) from a his-3 strain $\mathrm{G}_{g 59}$ were either irradiated with ultraviolet to a survival of ro per cent. and plated on selective media (minimal and "conidiating 
complete"; Grigg and Sergent, I96I), or plated directly as an unirradiated control. The his ${ }^{+}$colonies which appeared were sub-cultured on minimal or on complete medium. Later, small conidial inoculations were tested for growth behaviour on minimal, complete and histidine medium at $25^{\circ}$ and $35^{\circ} \mathrm{C}$. The cultures were retested after a period of approximately 4 weeks ("terminal " cultures). At the same time the first subcultures were transferred to complete and histidine medium tube slopes, and were tested as soon as macroconidia had formed (c. 4 days). For further details of the stocks and methods employed see Grigg and Sergent (196r).

\section{RESULTS}

Fifty-nine of the cultures from both ultraviolet and control groups, detected because of their ability to grow on either of the selective media used (minimal or complete), were subcultured and tested. Only twenty-five colonies proved completely stable when retested several times over the test period of 4 weeks (Group I in table I). Fourteen of these twenty-five colonies were retested over a longer period and proved quite stable over a period of 2 years. The remaining thirty-seven showed distinct and permanent alterations in their growth characteristics. Some changed to a form where they were inhibited on complete or histidine media as is shown in series II, III and IV in table I. Two (series VIII) showed a change from slow growth on the test media to faster growth. A large group (fifteen in all) changed from wild type to his- (series V) and two from being able to grow on minimal to an inability to grow on either minimal, histidine or complete medium (series VI).

The growth characteristics of the cultures grouped in series $\mathrm{V}$ because they " reverted" to histidine dependence showed some variation at $t_{0}$. Some were slow growers on all test media, others were temperature sensitive and some were even histidine inhibited. They all finished up as histidine dependent cultures. In instances where duplicate subcultures were taken from the original colony on the selection medium the type of change which occurred was always identical in the duplicates.

Apart from the instability of the growth characters there was also variation in the characteristics of the first-subcultures in other groups as well as group $\mathrm{V}$. The growth habits ranged from normal wild type growth on the three test media at both $25^{\circ}$ and $35^{\circ}$ to slow growth on all media or no growth on complete medium at $35^{\circ}$ or at both $35^{\circ}$ and $25^{\circ}$. Although these differences may be due to subcultures being at different stages of transition from wild type to some non-wild type character, an examination of stable wild types (Grigg and Sergent, I96I) from similar intervals suggests that the initial variation in wild type colony subcultures may reflect real intrinsic genetic differences between them.

What is the cause of the instability? There seem two obvious explanations.

I. Prototrophic heterokaryons have changed to non-prototrophic homokaryotic ones.

2. The prototrophs involved the labile attachment of episomic factors to either the $h^{-}$or suppressor of $h^{-}$genes.

At the time testing was carried out, most or all of the macroconidia to be produced by the small-tube cultures had been formed. That some selective 
process during growth or during conidial formation was responsible for the changed character of the subcultures seems unlikely.

A change of the existing conidial population from a heterokaryotic to a homokaryotic condition could occur by either a differential longevity of

TABLE :

Growth characteristic of prototrophic cultures tested immediately they first arose $\left(\mathrm{t}_{0}\right)$ and one month later $\left(\mathrm{t}_{1}\right)$

Growth behaviour

\begin{tabular}{|c|c|c|c|c|c|c|c|c|}
\hline \multirow{2}{*}{ Group } & \multirow{2}{*}{$\begin{array}{l}\text { Time of } \\
\text { Testing }\end{array}$} & \multicolumn{3}{|c|}{$25^{\circ}$} & \multicolumn{3}{|c|}{$35^{\circ}$} & \multirow{2}{*}{$\begin{array}{l}\text { No. of } \\
\text { colonie } \\
\text { tested }\end{array}$} \\
\hline & & Minimal & Complete & Histidine & Minimal & Complete & Histidine & \\
\hline I & $\begin{array}{l}t_{0} \\
t_{1}\end{array}$ & + & $\begin{array}{l}+ \\
+\end{array}$ & $\begin{array}{l}+ \\
+\end{array}$ & $\begin{array}{l}+ \\
+\end{array}$ & + & $\begin{array}{l}+ \\
+\end{array}$ & 25 \\
\hline II & $\begin{array}{l}t_{0} \\
t_{1}\end{array}$ & + & \pm & + & + & \pm & + & ? \\
\hline III & $\begin{array}{l}t_{0} \\
t_{1}\end{array}$ & + & + & \pm & + & + & \pm & \} \\
\hline IV & $\begin{array}{l}t_{0} \\
t_{1}\end{array}$ & $\begin{array}{l}+ \\
+\end{array}$ & \pm & \pm & + & \pm & \pm & $7-$ \\
\hline V & $\begin{array}{l}t_{0} \\
t_{1}\end{array}$ & $\begin{array}{r}* \\
+ \\
+ \\
+ \\
+ \\
-\end{array}$ & $\begin{array}{c}* \\
+ \\
+ \\
\pm \\
+\end{array}$ & $\begin{array}{l}* \\
+ \\
+ \\
+ \\
+ \\
+\end{array}$ & $\begin{array}{r}* \\
\\
+ \\
* \\
- \\
-\end{array}$ & $\frac{*}{ \pm}+$ & $\begin{array}{l}* \\
+ \\
+ \\
+ \\
+\end{array}$ & 15 \\
\hline VI & $\begin{array}{l}t_{0} \\
t_{1}\end{array}$ & \pm & \pm & \pm & \pm & \pm & \pm & \} \\
\hline VII & $\begin{array}{l}t_{0} \\
t_{1}\end{array}$ & $\begin{array}{l}+ \\
+\end{array}$ & $\bar{t}$ & + & + & $\bar{t}$ & + & $j$ \\
\hline VIII & $\begin{array}{l}t_{0} \\
t_{1}\end{array}$ & + & $\bar{t}$ & + & $\begin{array}{c}*+ \\
+\end{array}$ & $\bar{t}$ & $\begin{array}{c}*+ \\
+\end{array}$ & is \\
\hline & & & & & & & Total & 59 \\
\hline
\end{tabular}

+ Wild type growth rate.

* Intermediate growth rate (c. $0.5 \times$ wild type rate).

* Slow growth rate $(c .0 \cdot I \times$ wild type rate):

- No growth.

homokaryotic versus heterokaryotic ones, or generally of auxotrophic versus prototrophic conidia. Another possibility is that one karyotype survived longer than the other even when in the same cytoplasm.

Prototrophic conidia do die before mutant ones (see for example Pontecorvo, 1953). If this is the explanation of the instability one would expect that the viable population should consist of a mixture of two types of conidia in the case where the original prototrophic heterokaryon contained two non-prototrophic karyotypes (see for example stable heterokaryon A6-C in Grigg and Sergent, I96I). This may be the reason for the aberrant 
growth response on " complete" medium of a few of the histidine requiring cultures (series V) which arose from unstable prototrophic ones. Complete medium does not support the growth of his- $3 \mathrm{G}_{g 59}$ (or of any other histidine strains of Neurospora for that matter), but conidial inoculations from some series $\mathrm{V}$ cultures grew on both histidine and complete media and not on minimal. This is understandable if the population were heterogeneous for a histidine requiring and a complete requiring component.

If the decreased longevity of prototrophic conidia compared with mutant ones is evoked to explain the instability phenomenon one might wonder why the same thing did not happen to the stable and heterokaryotic prototrophs which were maintained as stocks for a period of more than 2 years. Even though the particular nature of the auxotrophic character is important in determining its relative longevity with respect to a prototroph (MacDonald and Pontecorvo in Pontecorvo, 1953) this scarcely seems to explain sufficiently the discrepancy.

As with many other instances of gene instability (for a recent summary see Dawson and Smith Keary, I963) the instability reported here may be due to the attachment of episomic elements to either the his or a suppressor, (su-his) site with the latter fitting the data best. If the episome factors detached from the su-his site without attaching to other sites in most of the cells then a change of character from $t \rightarrow h$ s $^{-}$would result. Attachment to another site would lead to "switch" mutations (Kaplan, 196I; Grigg and Sergent, I961) and would explain why many of the unstable prototrophic cultures changed to non-histidine mutant phenotypes, for example those of group III, IV and VI of table I.

Perhaps neither hypothesis ( 1 or 2 above) is mutually exclusive and while the original unstable prototrophs may be the result of the intervention of an episome-like factor the complete loss of the prototrophic character in all the cells of a single colony may be the consequence of differential survival of the non-prototrophic cells over the prototrophic ones.

\section{SUMMARY}

The growth behaviour of a number of prototrophic back-mutants of a histidine strain of Neurospora was observed over a period of a month, commencing when the prototrophs first arose. A majority of the cultures proved unstable and lost their prototrophic character. The possible causes of this instability are discussed.

\section{REFERENCES}

DAWSON, G. W. P., AND SMITH-KEARY, P. F. 1963. Episomic control of mutation in Salmonella typhimurium. Heredity, $18,1-20$.

GRIGG, G. W., AND SERGENT, D. I96I. Compound loci and coincident mutation in Neurospora. Zeit.f. Vererb., 92, 380-388.

KAPLAN, R. 1961. Spontaneous mutation von einer monoauxotrophic zu einer andrerer in einer schuft. (Auxotrophusprungmutation.) Zeit. f. Vererb., 92, $21-27$.

Pontecorvo, G. 1953. The genetics of Aspergillus nidulans. Advances in Genetics, 5, $14^{1-23^{8}}$. 